\title{
The lagrangian formalism for the derivation of Vlasov and Liouville equations on the (co)tangent bundles
}

\section{Opinion}

At the present time, the Lagrangian formalism can be recognized as a key technique for constructing cosmological models and investigating the general relativistic properties of the evolution of complex systems (including multiparticle ensembles and electromagnetic and scalar fields as subsystems). In fact, the study of each of these models is reduced to the construction of an action of a sufficiently general form containing terms that can later be interpreted as peculiar generators of terms in the resulting "equations of motion" of the system under consideration. However, in this case there are certain subtleties associated with the correct account of the structure of the terms in the action associated with the nonequilibrium nature of the evolution of the interacting many-particle systems in the gravitational field. In the overwhelming majority of methodological manuals on dynamics in the theory of relativity, the analysis is limited to the hydrodynamic level of motion (approximation of a continuous medium). At the same time, a complex structure (corpuscular, stellar clusters, etc.) of the general-relativistic system under investigation is actually ignored. Further, the structure of individual members in the total action should be investigated separately in order to exclude from consideration mathematically correct, but physically poorly grounded consequences; here, of course, a certain balance of the situation must be observed, since the "nonphysical" nature of the resulting equations may indicate the existence of fundamentally new effects that are in contradiction with existing paradigms.

And, finally, the need to optimize the formalism used in relation to specific tasks - here it is implied that the mathematical apparatus for analyzing the dynamics of a multiparticle system can be rationally adapted on the basis of the a priori set of physical limits of the problem being solved. In principle, to solve the problem of interpretation of the influence of the $\Lambda$-term in the Einstein equations in terms of the electromagnetic interaction included in the total Lorentz action with allowance for the subactions Einstein-Maxwell, etc., one can in principle use the ADM method; However, it is much simpler and more clearly to obtain canonical equations and write down the corresponding Vlasov-type equation directly. It is quite obvious that the dynamical properties obtained directly or indirectly depend on the structure of the kinetic potential (Lagrangian) of the system, such as evolution as a whole and a change in the state of partial properties (changes distributions of particles, geometric dimensions of the system and its allocated parts, accounting necessity of introduction or exclusion of types of interparticle interaction on the appropriate scales, etc.). However, even in the simplest case of a system of point neutral particles that have a mass in an external gravitational field are sufficient.

It is not easy to construct a single Lagrangian whose dynamic consequences can be called physically sound and relevant to reality. In fact, practically in all the manuals on GTR this issue is not raised, and the authors a priori take some definite form of the Lagrangian density as a single-valued "generating function" for establishing the form of the equations of motion for study of system dynamics. It is implicitly assumed that the main criterion for the legitimacy
Volume 3 Issue 2 - 2019

\author{
Nikolay Nikolaevich Fimin \\ Keldysh Institute of Applied Mathematics, Russia
}

Correspondence: Nikolay Nikolaevich Fimin, Keldysh Institute of Applied Mathematics, Russian Federation, I25047, Moscow, Miusskaya square, 4, Russia, Tel +79258877093 , Email oberon@kiam.ru

Received: March 30, 2018 | Published: March II, 2019

of the internal structure of the analyzed action is the possibility of obtaining on its basis the Einstein equations with the right-hand side containing the energy-momentum tensor of matter distributed in a certain way in the space-time region under study. Einstein's equations for the gravitational field due to a certain distribution of material objects (particles), as is known, can be obtained from the principle of stationarity of action by varying the sum of the Einstein-Hilbert and particle systems. The summation over individual particles in the second term of summary action can be replaced by integrating over an invariant (in the case of transformations of all components) volume element in an 8-dimensional (co) tangent bundle over a 4-dimensional configuration manifold along geodesics by means of the identity transition including the particle distribution density. Obviously, here there is ambiguity in the choice of the structure of the action, connected with the choice of the variables of the distribution function (the dependence on the velocity or momentum, which leads to the need to consider dynamics on the tangent or on the cotangent bundle) and an element of the 8-dimensional phase space: dimensional spacetime manifold is geometrically the only acceptable choice (providing a standard form of Einstein's field equations in the analysis of the Einstein-Hilbert action), then (co)tangent bundle 8-volume element generally depends on the type of "full-naturally induced" metric defined by quadratic (Pfaffian) forms. ${ }^{1}$

The use of the " full " (pseudo) metric on the bundle ( I + II , II , II + III, I + III) can be dictated only additional conditions for a specific physical task (for some classes of which it is necessary to use other types of metricization of phase space, such as the synectic metric, the Wilms metric, etc.). In the literature, an implicit agreement on the choice as a standard (or, rather, " demonstration ", because the a priori physical prerequisites of its advantages over others do not exist) type of the metric of the case I + III, i.e. the metric of the diagonal lift (Sasaki metric for tangent bundle, Tondeur-Sato metric for cotangent bundle). ${ }^{2}$ However, even for the simplest case a transition from a 4-dimensional configuration manifold to an 8-dimensional one (the space of support elements) ${ }^{3}$ is necessary. It turns out that the cosmological lambda-term in the Einstein equations is a consequence of this transition, and is the averaged curvature operator of an 8-dimensional configuration space.

\section{Acknowledgments}

None. 


\section{Conflicts of interest}

The author declares there is no conflict of interest.

\section{References}

1. Yano K Ishihara S. Tangent and cotangent bundles. Marcel Dekker, New York, USA. 1973;7(2):218-219.
2. Sasaki S. On the differential geometry of tangent bundles of Riemannian manifolds. Tohoku Mathematical Journal. 1958;10(3):338-354.

3. Vlasov AA. Statistical Distribution Functions. Nauka, Moscow (in Russian). 1966. 